\title{
Correção cirúrgica da esotropia em portadores de fixação excêntrica
}

\author{
Surgical correction of esotropia in eccentric fixation patients
}

\author{
Andréa Alejandra González Martinez ${ }^{1}$ \\ Mauro Goldchmit ${ }^{2}$ \\ Gustavo Bueno Camargo ${ }^{3}$ \\ Carlos Souza-Dias ${ }^{4}$
}

Trabalho realizado na Faculdade de Ciências Médicas da Santa Casa de Misericórida de São Paulo. São Paulo (SP).

${ }^{1}$ Ex-aluna do curso de especialização do serviço de oftalmologia da Faculdade de Ciências Médicas da Santa Casa de Misericórdia de São Paulo. São Paulo (SP).

${ }^{2}$ Assistente do serviço de oftalmologia da Faculdade de Ciências Médicas da Santa Casa de Misericórdia de São Paulo. São Paulo (SP).

${ }^{3}$ Aluno do curso de aperfeiçoamento da Faculdade de Ciências Médicas da Santa Casa de Misericórdia de São Paulo. São Paulo (SP).

${ }^{4}$ Chefe do serviço de estrabismo da Faculdade de Ciências Médicas da Santa Casa de Misericórdia de São Paulo. São Paulo (SP).

Endereço para correspondência: Andréa Alejandra González Martinez - Rua Thomé de Souza, 31 - São Bernardo do Campo (SP) CEP 09710-220

Email: andreateka@aol.com

Recebido para publicação em: 16.06.2004

Versão revisada recebida em 16.05.2005

Aprovação em 16.06.2005

Nota Editorial: Depois de concluída a análise do artigo sob sigilo editorial e com a anuência da Dra. Rosália Maria Simões Antunes Foschini sobre a divulgação de seu nome como revisora, agradecemos sua participação neste processo.

\begin{tabular}{|l|} 
RESUMO \\
\hline Objetivo: Avaliar o resultado da correção cirúrgica da esotropia em \\
pacientes com fixação excêntrica e compará-lo com o de pacientes esotró- \\
picos operados que não possuíam essa alteração sensorial. Métodos: \\
Estudo retrospectivo do resultado da correção cirúrgica da esotropia \\
essencial de 19 pacientes com fixação excêntrica do Serviço de Motilidade \\
Ocular Extrínsica da Santa Casa de São Paulo. Como grupo-controle, foram \\
estudados 17 pacientes esotrópicos com ambliopia estrábica e fixação \\
central e 16 pacientes esotrópicos sem ambliopia. O teste estatístico \\
utilizadofoi aplicação de variância para proporções (ANOVA). Resultados: \\
Nos 3 grupos estudados prevaleceu a subcorreção, 12 (63,2\%) casos no \\
grupo I, 13 (76,5\%) casos no grupo II e 13 (81,3\%) pacientes no grupo III. \\
O sucesso cirúrgico (desvios $\leq 10^{\Delta}$ ) ocorreu em 7 (36,8\%) pacientes do \\
grupo com fixação excêntrica, dos quais 4 casos eram de supercorreção e \\
3 de ortotropia. No grupo II, dos 7 casos de sucesso cirúrgico, 3 (17,6\%) \\
estavam com ortotropia e 1 (5,9\%) caso estava supercorrigido. No grupo \\
III, tivemos 5 (31,3\%) casos de sucesso cirúrgico, sendo 1 (6,3\%) de \\
ortotropia. Entre os 36 pacientes amblíopes, 5 (13,9\%) apresentaram \\
supercorreção. O erro padrão da média da correção cirúrgica foi de 4,6 no \\
grupo de pacientes com fixação excêntrica. O teste ANOVA para a média \\
da correção cirúrgica foi de p=0,349. Considerando o bom resultado \\
(desvio de até $10^{\Delta}$ a partir da posição primária do olhar), obtivemos um \\
p=0,847. Conclusão: A fixaçãoexcêntricanãorepresentou fator determinante \\
no resultado cirúrgico (bom vs mau) do desvio horizontal dos pacientes \\
com esotropia entre $20^{\Delta}$ e $50^{\Delta}$.
\end{tabular}

Descritores: Esotropia/cirurgia; Fixação ocular; Ambliopia; Estrabismo/fisiopatologia; Adaptação ocular; Análise de variância

\section{INTRODUÇ̃̃̃OO}

Os movimentos oculares induzidos por excitações retínicas são chamados reflexos optomotores, dentre os quais os principais são os reflexos de fixação, fusão e seguimento. O reflexo de fixação é a habilidade do indivíduo, sob condições fisiológicas, em manter a imagem de um objeto, sobre a qual a atenção é direcionada, na fóvea ${ }^{(1-2)}$. A fixação foveal incorpora a sensação espacial de estar olhando no centro do sistema subjetivo visual ${ }^{(2)}$. É um processo dinâmico, mantido através de um mecanismo de retroinformação ${ }^{(2-3)}$.

A fixação em recém-nascidos, ainda que rudimentar, surge ao final do primeiro mês de vida, alcançando seu refinamento no quarto mês, juntamente com o desenvolvimento foveal ${ }^{(1)}$. 
Com o aparecimento de um desvio ocular, ocorre supressão da fóvea do olho desviado e células retínicas extrafoveais podem assumir um valor espacial "em frente", substituindo a hierarquia funcional da fóvea ${ }^{(2)}$. Assim, surge a mais grave perturbação sensorial do estrabismo, a fixação excêntrica ${ }^{(1)}$. Trata-se de um fenômeno monocular ${ }^{(4)}$ e está presente em $30 \%$ dos casos de estrabismo com ambliopia ${ }^{(5)}$. Está sempre acompanhada de correspondência retínica anômala.

Entre os métodos de investigação da fixação excêntrica, o mais utilizado é a visuscopia de Cüppers ${ }^{(6)}$. Com esse exame, a fixação pode ser classificada em central ou excêntrica, podendo esta ser parafoveal, paramacular, interpapilomacular ou periférica ${ }^{(1)}$.

Não encontramos relatos na literatura quanto ao resultado da correção cirúrgica da esotropia em pacientes portadores de fixação excêntrica. O presente estudo teve como objetivos avaliar o resultado da correção cirúrgica da esotropia nesses pacientes e compará-lo com o de pacientes esotrópicos operados que não possuem fixação excêntrica.

\section{MÉTODOS}

Avaliaram-se retrospectivamente os resultados da correção cirúrgica da esotropia essencial em 19 pacientes portadores de fixação excêntrica (Grupo I), diagnosticada com o visuscópio de Cüppers, pertencentes ao setor de Motilidade Ocular Extrínseca do Departamento de Oftalmologia da Santa Casa de Misericórdia de São Paulo, operados no período de 1980 a 2002. Como grupos controles, foram estudados os resultados cirúrgicos de 17 pacientes esotrópicos com ambliopia estrábica e fixação central, confirmada através da visuscopia (grupo II) e 16 pacientes esotrópicos sem ambliopia (Grupo III). Considerouse ambliopia a diferença de acuidade visual entre os olhos maior que 2 linhas na tabela de Snellen, com a melhor correção óptica.

Os critérios de inclusão foram: 1) esotropia comitante de $20^{\Delta}$ a $50^{\Delta}$; 2) operação monocular; 3) ausência de cirurgia prévia de estrabismo; 4) ausência de incomitância longe-perto; 5) ausência de anisotropia do meridiano vertical ou disfunção de músculos oblíquos; 6 ) equivalente esférico $\leq 3,5$ dioptrias esféricas positivas; 7) ausência de anisometropia $\geq+3,5$ dioptrias esféricas positivas; 8)ausência de DVD; 9) ausência de nistagmo; 10) ausência de atraso do desenvolvimento neuropsicomotor; 11) ausência de alterações orgânicas oculares e 12) seguimento pós-operatório mínimo de 3 meses.

Todos os pacientes foram submetidos a avaliação oftalmológica, que constou de exame externo, medida da acuidade visual, biomicroscopia, refratometria e fundoscopia. No exame da motilidade ocular extrínseca, realizou-se medida do ângulo do desvio na posição primária do olhar e nas supra, infra, dextro e levoversões, com a melhor correção óptica, por meio do teste de oclusão alternada com prisma ou pelo método de Krimsky, dependendo da capacidade de fixação do paciente e da acuidade visual.

A acuidade visual no olho amblíope com a melhor correção óptica no grupo I foi de movimentos de mão a 0,15 e, no grupo II, de 0,1 a 0,6. As informações com relação à idade e sexo encontram-se no quadro abaixo.

O planejamento cirúrgico nos três grupos baseou-se na magnitude do desvio na posição primária do olhar. O retrocesso do músculo reto medial em milímetros foi, em média, de $4,9 \pm$ $0,5(4,0$ a 6,0$)$ no grupo I, $5,0 \pm 0,3(4,0$ a 6,0$)$ no grupo II e 4,8 \pm $0,3(4,0$ a 5,5$)$ no grupo III. A ressecção média do músculo reto lateral foi de $6,4 \pm 1,2(4,0$ a 8,0$)$ no grupo I, $5,8 \pm 0,9(4,0$ a 8,0$)$ no grupo II e de $6,4 \pm 0,9(4,0$ a 8,0$)$ no grupo III.

O tempo médio de seguimento em meses foi de $16,9 \pm 14,3$ (3 a 110) para o grupo I; 11,9 $\pm 7,8$ ( 3 a 52) para o grupo II e 44,7 $\pm 44,7$ ( 3 a 192) para o grupo III.

Como critério de sucesso cirúrgico, ou de bons resultados, consideraram-se desvios $\leq 10^{\Delta}$, convergente ou divergente, a partir da posição de ortotropia.

Para a análise estatística da média das correções cirúrgicas e do resultado cirúrgico dos três grupos de pacientes (bom vs mau), utilizou-se o programa SPSS (Statistical Package for Social Science) em sua versão 10.0 e o teste utilizado foi Aplicação de Variância para Proporções (ANOVA) ${ }^{(6-8)}$. O nível de rejeição para nulidade foi fixado em valor menor ou igual a $0,05(5 \%)$.

Este estudo obteve aprovação do Comitê de Ética Médica da Faculdade de Ciências Médicas da Santa Casa de Misericórdia de São Paulo no dia 29/10/2003 (projeto nํ 019/03).

\section{RESULTADOS}

Os resultados cirúrgicos dos pacientes com e sem fixação excêntrica estão apresentados na tabela e no gráfico que se seguem.

A análise da tabela mostra que a supercorreção ocorreu em $3(15,8 \%)$ pacientes do grupo com fixação excêntrica e em 2 $(12,5 \%)$ pacientes do grupo sem ambliopia. O sucesso cirúrgico ocorreu em $7(36,8 \%)$ casos do grupo com fixação excêntrica, em $7(41,2 \%)$ do grupo com ambliopia sem fixação excêntrica e em $5(31,3 \%)$ pacientes do grupo sem ambliopia. Nos 3 grupos estudados prevaleceu a sub-correção, $12(63,2 \%)$ casos no grupo I, 13 (76,5\%) casos no grupo II e 13 (81,3\%) pacientes no grupo III.

Em nenhum dos pacientes estudados evidenciou-se variação de desvio maior que $10^{\Delta}$ no decorrer do período pósoperatório. Além disso, nenhuma variação observada provocou mudança quanto ao sucesso cirúrgico.

\begin{tabular}{|c|c|c|c|}
\hline \multicolumn{4}{|c|}{$\begin{array}{l}\text { Quadro. Idade e sexo dos pacientes esotrópicos com fixação } \\
\text { excêntrica (grupo I), com ambliopia e sem fixação excêntrica } \\
\text { (grupo II) e sem ambliopia (grupo III) submetidos a correção cirúr- } \\
\text { gica do estrabismo }\end{array}$} \\
\hline & $\begin{array}{c}\text { Grupo I } \\
N=19\end{array}$ & $\begin{array}{c}\text { Grupo II } \\
N=17\end{array}$ & $\begin{array}{c}\text { Grupo III } \\
N=16\end{array}$ \\
\hline Idade (anos) & $\begin{array}{c}23,8 \pm 7,0 \\
(7 \text { a } 45)\end{array}$ & $\begin{array}{c}17,5 \pm 7,8 \\
(5 \text { a } 45)\end{array}$ & $\begin{array}{c}11,9 \pm 5,6 \\
(4 \text { a } 33)\end{array}$ \\
\hline Sexo (masc/fem) & $13 / 6$ & $10 / 7$ & $6 / 10$ \\
\hline
\end{tabular}




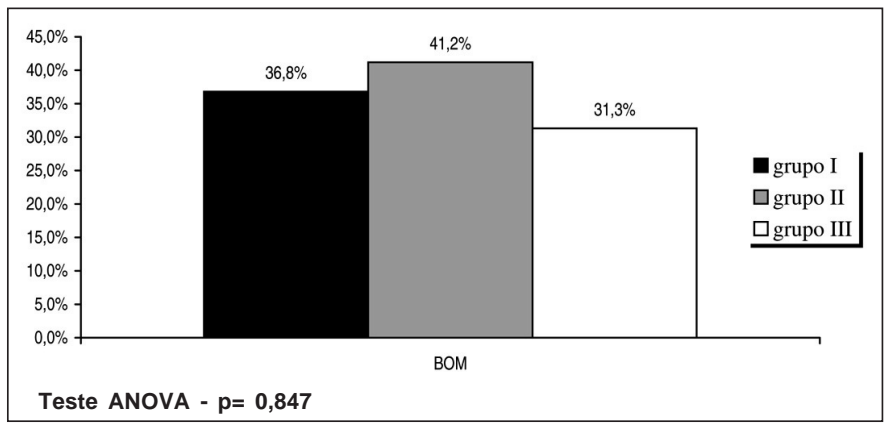

Gráfico - Resultado da correção cirúrgica segundo a presença de bons resultados dos pacientes esotrópicos com fixação excêntrica (grupo I), nos pacientes amblíopes sem fixação excêntrica (grupo II) e sem ambliopia (grupo III). Santa Casa /S.P./ 1980-2002

\section{DISCUSSÃO}

O presente estudo revelou que a subcorreção prevaleceu nos 3 grupos estudados. Esses resultados podem em parte ser justificados pela orientação que existe no setor de Motilidade Ocular Extrínseca do Departamento de Oftalmologia da Santa Casa, em que o planejamento cirúrgico tem como objetivo buscar a ortotropia e discreta subcorreção nos casos de ambliopia, pelo reconhecido papel que esta tem no desenvolvimento de supercorreções tardias.

Quando foram comparadas as médias das correções cirúrgi- cas em porcentagem (correção/pré-operatório x100) dos 3 grupos e aplicado o teste ANOVA, foi obtido um $\mathrm{p}=0,349$, ou seja, as porcentagens das correções cirúrgicas foram semelhantes.

O erro padrão médio da correção nos três grupos foi considerado grande ( $>2$, tabela) o que mostra a grande dispersão dos dados obtidos a partir da média, revelando a pouca previsibilidade dos resultados, principalmente do grupo com fixação excêntrica, no qual obtivemos o maior erro padrão médio $^{(4,6)}$.

O estudo de Prieto-Díaz revelou que $80 \%$ das supercorreções a longo prazo nas esotropias decorriam da hiperfunção dos músculos oblíquos, hipermetropia maior que 4,0 dioptrias esféricas, anisometropia, ambliopia incurável e cirurgias prévias para correção do estrabismo ${ }^{(9)}$. Entre os 36 pacientes amblíopes avaliados no presente estudo, $5(13,8 \%)$ apresentaram supercorreção no período médio de 12 meses de seguimento.

Sabe-se que o resultado pós-operatório da cirurgia para correção da esotropia pode modificar-se com o passar do tempo ${ }^{(10)}$. Uma vez que nos 3 grupos estudados não se evidenciou variação de desvio maior que $10^{\Delta}$ no período de seguimento pós-operatório médio de aproximadamente 12 meses, neste trabalho o resultado da correção cirúrgica, segundo a presença de bons resultados, não revelou diferença estatísti$\mathrm{ca}(\mathrm{p}=0,847)$.

Prieto-Diaz afirma que a cirurgia monocular proporciona não somente maior correção do ângulo de desvio, como também

\begin{tabular}{|c|c|c|c|c|c|c|c|c|c|}
\hline & \multicolumn{3}{|c|}{ Grupo I } & \multicolumn{3}{|c|}{ Grupo II } & \multicolumn{3}{|c|}{ Grupo III } \\
\hline & pré & pós & corr & pré & pós & corr & pré & pós & corr \\
\hline & 20 & 20 & 0 & 25 & 8 & 17 & 25 & 15 & 10 \\
\hline & 20 & 0 & 20 & 25 & 20 & 5 & 25 & -28 & 53 \\
\hline & 25 & 10 & 15 & 30 & 15 & 15 & 25 & 13 & 12 \\
\hline & 25 & 10 & 15 & 30 & 0 & 30 & 30 & 20 & 10 \\
\hline & 30 & 20 & 10 & 30 & 15 & 15 & 30 & 4 & 26 \\
\hline & 30 & -15 & 45 & 30 & 25 & 5 & 30 & 10 & 20 \\
\hline & 35 & -30 & 6 & 35 & 10 & 25 & 30 & 28 & 2 \\
\hline & 35 & 10 & 25 & 35 & 20 & 15 & 35 & 12 & 23 \\
\hline & 35 & -30 & 65 & 35 & -10 & 45 & 35 & 30 & 5 \\
\hline & 40 & 30 & 10 & 35 & 22 & 13 & 35 & 0 & 35 \\
\hline & 40 & 25 & 15 & 38 & 20 & 18 & 35 & 20 & 15 \\
\hline & 45 & 25 & 20 & 40 & 0 & 40 & 35 & -20 & 55 \\
\hline & 45 & 30 & 15 & 40 & 4 & 36 & 35 & 30 & 5 \\
\hline & 45 & 30 & 15 & 40 & 0 & 40 & 45 & 25 & 20 \\
\hline & 45 & 0 & 45 & 45 & 25 & 20 & 45 & 8 & 37 \\
\hline & 50 & 12 & 38 & 45 & 27 & 18 & 50 & 10 & 40 \\
\hline & 50 & -6 & 56 & 50 & 35 & 15 & & & \\
\hline & 50 & 0 & 50 & & & & & & \\
\hline & 50 & 15 & 35 & & & & & & \\
\hline $\bar{X}$ & 37,6 & 8,2 & 29,4 & 35,8 & 13,9 & 21,9 & 34,1 & 11,1 & 23,0 \\
\hline S & 10,3 & 18,6 & 19,9 & 7,1 & 12,1 & 12,1 & 7,4 & 16,4 & 16,7 \\
\hline \multicolumn{2}{|l|}{ E.P.M. } & 4,6 & & & 3,0 & & & 4,2 & \\
\hline
\end{tabular}


resultados mais previsíveis que a cirurgia dos 2 retos medias ${ }^{(9)}$. Trabalho realizado em 2000 demonstrou haver estabilidade dos resultados cirúrgicos das esotropias no período entre 1 mês e 1 ano pós operatório, tanto com recuo dos retos mediais como com retrocesso-ressecção monocular, razão pela qual adotouse para o presente estudo o seguimento mínimo de 3 meses $^{(11)}$. Estudo realizado por alguns autores, revelou que o sucesso da cirurgia para correção da esotropia tem-se mostrado por volta de $72,9 \%$, dado que não se repete em nosso estudo, que pode ser justificado pela amostra pequena de pacientes ${ }^{(12)}$. Pode contribuir o fato de que muitos dos pacientes foram operados por residentes em diferentes estágios de aprendizado, embora sempre acompanhados pelos assistentes do Departamento. No entanto, deve-se considerar também que os assistentes apresentam técnicas distintas para realizar um mesmo plano cirúrgico, o que pode também contribuir para os resultados pósoperatórios que obtivemos neste trabalho. Nota-se que o excedente de subcorreção, além de $10^{\Delta}$ estabelecidas, foi de $3,9^{\Delta}$ no grupo II e de $1,1^{\Delta}$ no grupo III, valores insignificantes na prática, mas que concorreram para a prevenção do surgimento da exotropia pós-operatória, tão comum nos pacientes operados anos após a operação.

\section{CONCLUSÃO}

Com o presente estudo, conclui-se que a fixação excêntrica não representou fator determinante no resultado cirúrgico (bom vs mau) do desvio horizontal dos pacientes com esotropia entre $20^{\Delta}$ e $50^{\Delta}$, operados no serviço de Motilidade Ocular Extrínseca da Santa Casa de São Paulo, em um período médio de 16,9 $\pm 14,3$ ( 3 a 110) meses.

\section{ABSTRACT}

Purpose: To evaluate the result of the surgical correction of esotropic patients with eccentric fixation and to compare it with operated on esotropic patients who did not have this sensorial disorder. Methods: A retrospective study of the result of the surgical correction of essential esotropia in 19 patients with eccentric fixation of the "Santa Casa de São Paulo". As group controls, 17 esotropic patients with strabismic amblyopia and central fixation and 16 esotropic patients without amblyopia who had been operated on. The statistical test was application of variance for proportions (ANOVA). Results: In the 3 studied groups undercorrection prevailed, $12(63,2 \%)$ cases in group I, $13(76,5 \%)$ cases in group II and $13(81,3 \%)$ patients in group III. The surgical success occurred in $7(36,8 \%)$ patients of the group with eccentric fixation, of whom 4 cases were overcorrected and 3 of orthotropia. In group II, of the 7 cases of surgical success, $3(17,6 \%)$ had orthotropia and $1(5,9 \%)$ case presented with overcorrection. In group III, we had $5(31,3 \%)$ cases of surgical success, $1(6,3 \%)$ of them with orthotropia. Among the 36 amblyopic patients, $5(13,9 \%)$ presented overcorrection. The standard error of the average of the surgical correction was 4,6 in the group of patients with eccentric setting. ANOVA test for the average of the surgical correction was $p=0,349$. Considering the good result (a deviation smaller than $10^{\Delta}$ ), the statistical analysis revealed a $p=0,847$. Conclusion: The eccentric setting did not represent a determinant factor in the surgical result (good versus bad) of the horizontal surgical alignment.

Keywords: Esotropia/surgery; Fixation, ocular; Amblyopia; Strabismus/physiopathology; Adaptation, ocular; Analysis of variance

\section{REFERÊNCIAS}

1. Prieto-Diaz J, Souza-Dias C. Função sensorial. In: Pietro-Diaz J, Souza-Dias C. Estrabismo. 4a ed. São Paulo: Santos; 1986. p.1-62.

2. Mackensen G. Diagnosis and phenomenology of eccentric fixation. Int Ophthalmol Clin. 1966;6(3):397-409.

3. Linksz A. Theory of pleoptics. Trans Am Acad Ophthalmol Otolaryngol. 1961;1(4):747-85.

4. Keiner GB. Physiology and pathology of optomotor reflexes. Am J Ophthalmol. 1956;42(2):233-49.

5. Priestlely BS. Pleoptic instruments and methods. Int Ophthalmol Clin. 1961;1(4):787-827.

6. Von Noorden GK. Pathogenesis of eccentric fixation. Am J Ophthalmol. 1966;61(3):399-421.

7. Doria Filho U. ANOVA e a estatística F. In: Introdução à bioestatística: para simples mortais. 5a ed. São Paulo: Negócio; 1999. p.115-8.

8. Spiegel MR. Noções de análise de variância. In: Assumpção MM, editor. Estatística. São Paulo: Makron Books; 1993. p.515-67.

9. Prieto-Díaz J. Cirugía monocular vs. cirugía binocular. Arch Oftalmol B Aires. 1976;51:287-91.

10. Maruo T, Kubota N, Iwashige H, Kamiya Y. Long-results afters strabismus surgery. Graefe's Arch Clin Exp Ophthalmol. 1988;226:414-7.

11. Pizza R. Estabilidade dos resultados cirúrgicos em esotropias [especialização]. São Paulo: Santa Casa de Misericórdia de São Paulo; 2000.

12. Keenan JM, Willshaw HE. The outcome of strabismus surgery in childhood esotropia. Eye. 1993;7(Pt 3):341-5.

\section{Ao enviar um artigo para publicação, leia ATENTAMENTE as instruções para autores, constante no final de cada fascículo.}

